\title{
Ultrasound-guided genicular nerve block versus physical therapy for chronic knee osteoarthritis: a prospective randomised study
}

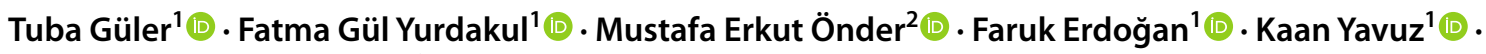

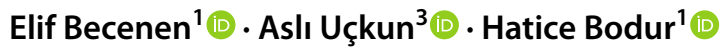

Received: 5 December 2021 / Accepted: 1 February 2022 / Published online: 14 February 2022

(c) The Author(s), under exclusive licence to Springer-Verlag GmbH Germany, part of Springer Nature 2022

\begin{abstract}
To compare the effectiveness of ultrasound-guided genicular nerve block (GNB) and physical therapy (PT) in patients with chronic knee osteoarthritis. A prospective randomised study with 102 patients (45-70 years) was performed wherein the patients received ultrasound-guided GNB $(n=51)$ and PT $(n=51)$ along with a standard home exercise programme. Scores for pain on a Visual Analogue Scale (VAS), Western Ontario and McMaster Universities Osteoarthritis Index (WOMAC) and 6-min walking test (6MWT) were assessed pre-treatment and at 2- and 12-weeks post-treatment. Both groups were comparable for sociodemographic characteristics. VAS scores (in $\mathrm{mm}$ ) in the ultrasound-guided GNB group at 0,2 and 12 weeks were $7.01 \pm 1.36 ; 3.71 \pm 2.18 ; 5.08 \pm 2.22(\mathrm{p}<0.001)$ and $6.64 \pm 1.99 ; 4.35 \pm 1.09 ; 5.25 \pm 1.33,(p<0.001)$ in the PT group. While the increase in the 6MWT test in the 2 nd week was similar for both groups $(p=0.073)$, the increase in walking distance was greater in the ultrasound-guided GNB group at 12 weeks $(p=0.046)$. As compared to PT, ultrasound-guided GNB is beneficial in reducing pain and increasing functional and physical capacity, with greater retention of effects on the physical capacity seen at 12 weeks. Trial registration number: ClinicalTrials.gov (NCT04782401).
\end{abstract}

Keywords Knee joint $\cdot$ Nerve block $\cdot$ Osteoarthritis $\cdot$ Physical therapy modalities $\cdot$ Ultrasound

Tuba Güler

tubakulu@yahoo.com

Fatma Gül Yurdakul

fatmagulonder@gmail.com

Mustafa Erkut Önder

erkutonder@hotmail.com

Faruk Erdoğan

frkerd25@gmail.com

Kaan Yavuz

kaanyvz@yahoo.com

Elif Becenen

ebecenen@gmail.com

Aslı Uçkun

draslical@gmail.com

Hatice Bodur

haticebodur@gmail.com

1 Physical Medicine and Rehabilitation Hospital, Ankara City Hospital, Ankara, Turkey

2 Division of Rheumatology, Training and Research Hospital, Aksaray University, Aksaray, Turkey

3 Department of Physical Medicine and Rehabilitation, İzmir Medicana Hospital, İzmir, Turkey

\section{Introduction}

Knee osteoarthritis (KOA) is a chronic joint disease that leads to pain, disability and impaired quality of life, resulting in significant social and healthcare detriments worldwide [1-3]. Furthermore, these public and economic implications associated with KOA are expected to increase in the future $[1,2]$. With a global increase in the proportion of the elderly population, general obesity rates and consequently, the incidence of KOA, clinicians are now focussing on novel treatment strategies [1]. Currently, both non-surgical and surgical interventions are used for managing KOA [1, 2]. Non-surgical options include patient education, self-management strategies, weight loss, physical therapy (PT), bracing or foot orthosis, oral analgesics, non-steroidal anti-inflammatory drugs, steroids, hyaluronic acid, platelet-rich plasma injections, prolotherapy, stem cell therapy and genicular nerve blocks [1, 4], all of which aim to relieve pain, improve function and postpone the requirement for surgery.

PT is an established and evidence-based treatment option to reduce pain and improve function in $\operatorname{KOA}[5,6]$ with the use of PT modalities, such as thermal treatments, 
therapeutic ultrasound, electrical stimulation and laser therapy, which are known to modulate inflammation, while being effective on pain, function and quality of life [6, 7].

On the other hand, genicular nerve block (GNB) is a recently developed therapeutic option for KOA that targets the three sensory nerves of the knee: superior lateral, superior medial and inferior medial genicular nerve, to inhibit pain transmission to the central nervous system [4, 8]. Only a few studies have been conducted using GNB in patients with chronic KOA which revealed a reduction in pain and improvement in knee function [9-12]. Eventually, GNB gained popularity in the field of rheumatology to modulate inflammation in patients with rheumatoid arthritis [13]. Studies investigating the different methods of administering GNB, i.e. ultrasound-guided versus fluoroscopy-guided GNB, in chronic KOA have reported no difference in treatment efficacy between the two methods. However, Kim et al. [12] stated that ultrasound is more favourable for imaging since it does not require radiation exposure.

Although both GNB and PT are beneficial in patients with KOA, to our knowledge, no study has compared the effectiveness of the two interventions. Therefore, this study aims to compare the effects of ultrasound-guided GNB and PT on pain and functional status in patients with chronic KOA.

\section{Materials and methods}

\section{Study design}

This single-blinded, prospective, randomised study was conducted at the Ankara City Hospital-Physical Medicine and Rehabilitation outpatient clinic, between March 2020 and February 2021. This study was approved by the Local Ethics Committee (E-18-1968) and conducted in accordance with the Declaration of Helsinki; informed consent was obtained from all participants. The trial was registered with ClinicalTrials.gov (NCT04782401).

\section{Study participants and procedure}

The patients aged 45-70 years diagnosed with KOA using the American College of Rheumatology criteria [14] and having Kellgren-Lawrence grade 2 and 3 radiological osteoarthritis [15] were enrolled in the study. Patients were excluded if they had received a glucocorticoid or hyaluronic acid injection, or oral glucosamine, or had undergone PT for knee pain within the last 6 months, besides a history of inflammatory arthritis, knee surgery, existing neurological or mental disorders and pacemaker use.

\section{Randomisation}

Sociodemographic characteristics (age, gender, body mass index (BMI), education level) of all patients were recorded. Then, they were randomised into two groups by an independent researcher using a covariant-focussed randomisation/minimisation method, in which the researcher specified stratification according to factors (age and sex) that may affect the intervention outcomes. The patients were then assigned to intervention groups using computer-generated randomisation of participant numbers.

The ultrasound-guided injections were administered by a single physician having an experience of $>5$ years in ultrasonography. Additionally, both groups received a standard home exercise programme (detailed below). The outcome measures were assessed by two independent physicians, each evaluating one of the groups, who were not involved in the randomisation or treatment stages. The assessments were performed at three time points: before the treatment, 2 weeks and 12 weeks after the treatment.

\section{Interventions}

\section{Ultrasound-guided GNB}

We used a $12 \mathrm{MHz}$ linear probe (Logiq 9, General Electric, Milwaukee, WI) for the intervention. The patient lay supine with a pillow under the ipsilateral popliteal fossa to visualise the injection site comfortably. A $6 \mathrm{ml}$ solution-mixing $5 \mathrm{ml}$ of $2 \%$ lidocaine and $1 \mathrm{ml}$ of $40 \mathrm{mg}$ triamcinolone, was injected under ultrasound guidance using 23 $\mathrm{G}(0.6 \times 60 \mathrm{~mm})$ needles into the superomedial, inferomedial and superolateral genicular nerve branches equally.

The intervention was carried out as described by Yilmaz et al. [16]. First, the femoral epicondyle was determined by the transducer; then, the genicular arteries running in this periosteal region were projected and verified by colour Doppler flow (Fig. 1). Our injection points were the branches of the genicular nerve associating with the superomedial, inferomedial and superolateral branches of the genicular artery. The in-plane technique was preferred for best visualisation during injections (Fig. 2).

\section{Physical therapy}

In the PT group, all interventions were given with the patient in supine and knees extended. First, the superficial heating was applied using a moist hot pack for $20 \mathrm{~min}$, followed by ultrasonic therapy using a $3 \mathrm{~cm}^{2}$-headed transduce in continuous mode at $1 \mathrm{MHz}$ frequency and $1.5 \mathrm{Watt} / \mathrm{cm}^{2}$ for $5 \mathrm{~min}$ (Eletronica Pagani, Paderno Dugnano, Italy) in a rounding manner to achieve deep thermal effects. Next, conventional transcutaneous electrical nerve stimulation was applied 


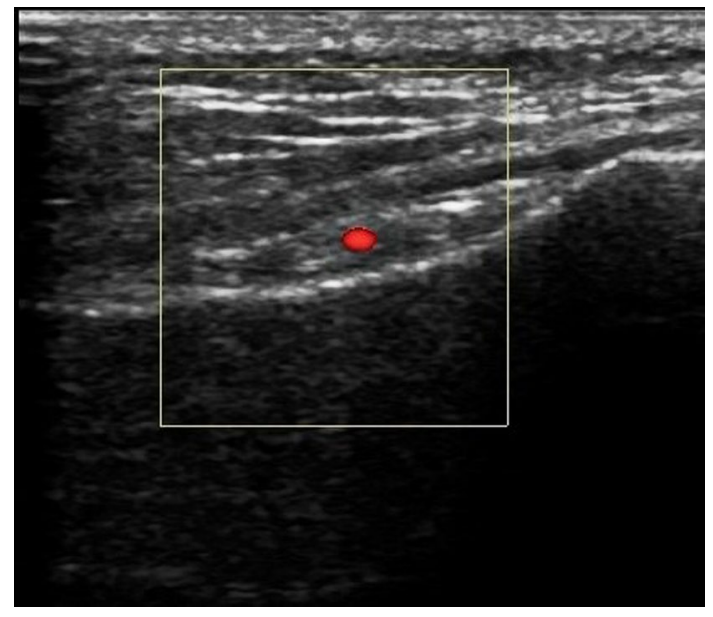

Fig. 1 Sonographic image of the inferomedial genicular artery

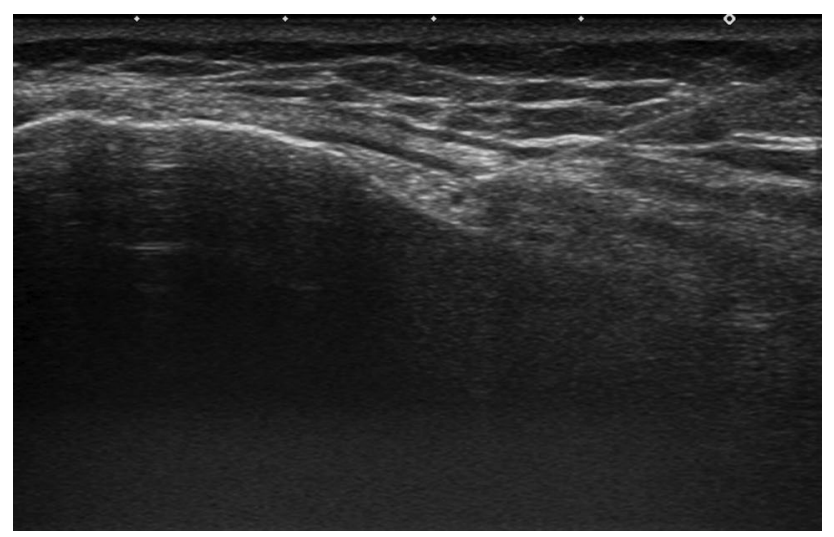

Fig. 2 The in-plane needle for inferomedial genicular nerve block

(TENScare, Surrey, United Kingdom) with 4 surface electrodes of 5-7 cm size each surrounding the knee (2 above and 2 below the patella), with a maximum $100 \mathrm{~Hz}$ pulse, for $20 \mathrm{~min}$. The intensity was adjusted as per the patient's tolerance limit. A total of 10 PT sessions were given for 5 days a week by the same physician.

\section{Exercise programme}

Both groups received an additional home exercise programme, including isometric and isotonic quadriceps strengthening, hamstring stretching and knee range-ofmotion exercises 10 repetitions of each exercise in a session, 3 sessions a day, 3 days a week. All exercises were demonstrated to the patient in detail.

The patient was instructed to put a rolled towel at the back of the knee in a sitting position with the knee extended, then compress the towel forcing the knee into hyperextension for $5 \mathrm{~s}$ and release serially for isometric quadriceps strengthening. Next, the patient lay supine and raised the involved leg for $10 \mathrm{~cm}$ above the bed in a straight position holding for $5 \mathrm{~s}$, and moved back down. For the isotonic quadriceps strengthening, the patient was instructed to straighten the knee into full extension while sitting and holding for $5 \mathrm{~s}$, and then flex the knee to $90^{\circ}$ serially, in the first week. In the second week, the patient performed the same exercise with $0.5 \mathrm{~kg}$ weight tied to the ankle. Active hamstring stretching was performed by flexing the ankle in a dorsal position while flexing the trunk in a sitting position. For the range-of-motion exercises, the patient was instructed to flex and extend the knee in the maximum tolerable ranges serially [17].

\section{Primary outcome measurement}

\section{Pain assessment}

A 10-mm Visual Analogue Scale (VAS), where $0 \mathrm{~mm}$ indicated no pain, and $10 \mathrm{~mm}$ indicated severe pain [18] was used to record the knee pain; additionally, the duration of pain was also recorded.

\section{Secondary outcome measurement}

\section{Physical function}

The Western Ontario and McMaster Universities (WOMAC) index was used to evaluate knee functionality. It consists of three subscales: Pain (5 questions), stiffness (2 questions) and function (17 questions). The total scores range from 0 to 96 , with higher scores indicating poor functionality. The validity and reliability of the WOMAC index have been demonstrated in Turkish patients with KOA [19].

\section{Physical capacity}

The 6-min walk test (6MWT) was used to evaluate physical capacity. Patients were asked to walk on a $30-\mathrm{m}$ corridor marked with 3-m intervals for $6 \mathrm{~min}$ at their preferred speed; rest or alteration in walking speed was allowed if needed. The maximum distance covered in 6 min throughout the 30-m corridor was measured [20].

Our primary end point was to achieve a decrease of $40 \%$ in overall VAS score at second week.

As side effect parameters, it was questioned whether there was new-onset pain, swelling, redness, itching or any discomforting feeling in the treated area for both groups.

\section{Statistical analysis}

Power analysis was conducted by $\mathrm{G}^{*}$ power version 3.0.10 to estimate the sample size. To gain the power of $0.80(\alpha$ 
(Type I error) $=0.05$ ), a sample size of 51 was obtained for each group. Effect size calculated according to our pilot study as 0.23 (Baseline-second week VAS mean and SD values were used).

All analyses were carried out using SPSS version 23.0 (IBM Corporation, Armonk, New York, USA). Normality was tested using the Shapiro-Wilk test and the continuous data were reported as mean and standard deviation or median (minimum-maximum). The two groups were compared using Student's $t$-test or Mann-Whitney $U$ test. Categorical variables were reported as frequencies and percentages. Outcomes at baseline and the two follow-up points were analysed using two-way repeated-measures analysis of variance or Friedman test. A $p$ value of $<0.05$ was considered for statistical significance and the $95 \%$ confidence intervals were calculated.

\section{Results}

A total of 102 patients-51 in each group, were enrolled in the study (GNB group - mean age $=55.88 \pm 7.62$ years; Female: Male $=40: 11 ;$ PT group —mean age $=53.01 \pm 9.22$ years; Female: Male: 41:10). Eleven patients in the GNB group and five patients in the PT group did not attend follow-up controls, therefore, post-treatment analyses were completed for 40 patients in the ultrasound-guided GNB group and 46 patients in the PT group. Figure 3 presents the flowchart of study participants. Both groups were comparable in terms

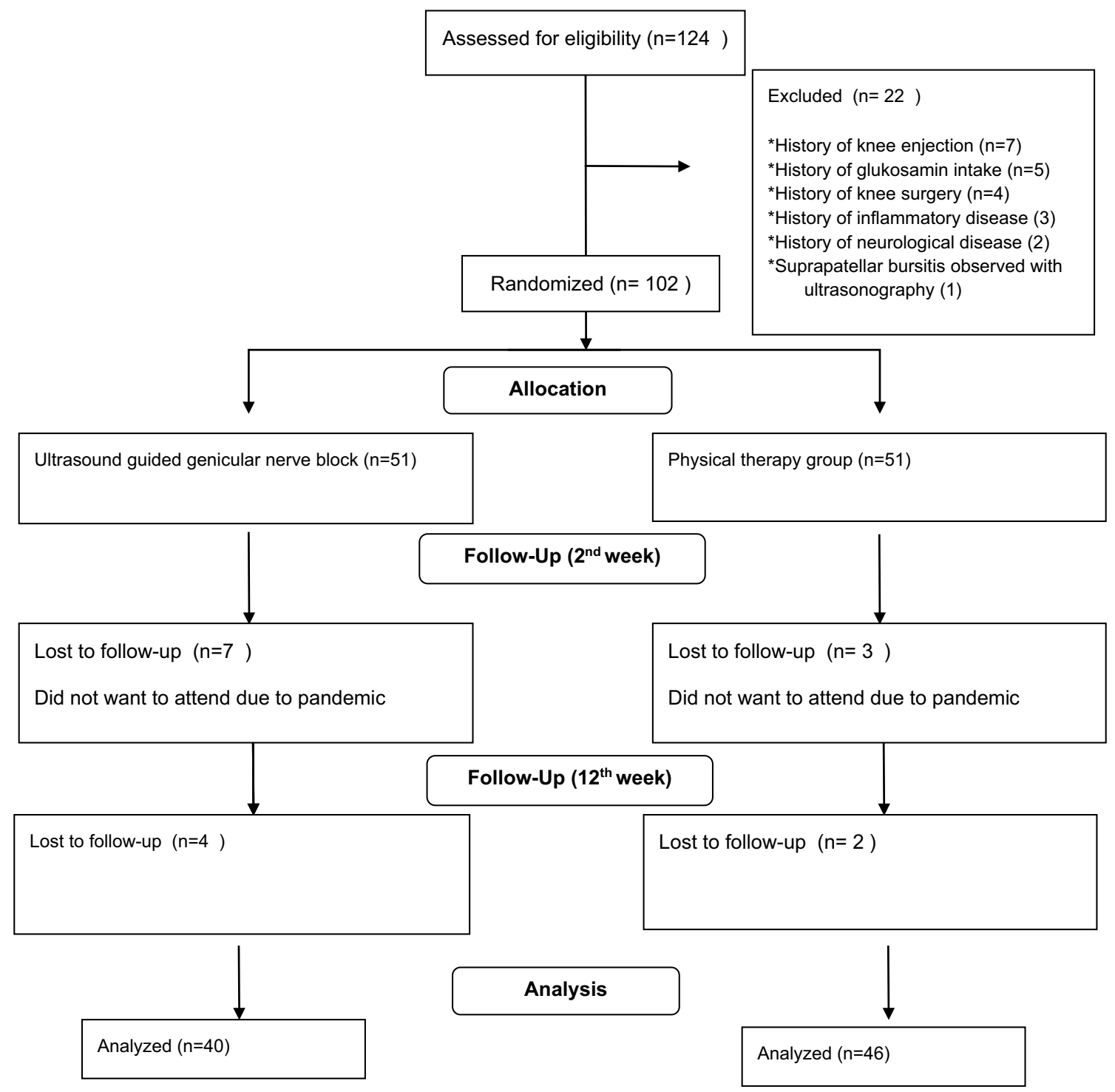

Fig. 3 Flowchart of the study participants 
Fig. 4 Improvement in Visual Analogue Scale (VAS) scores

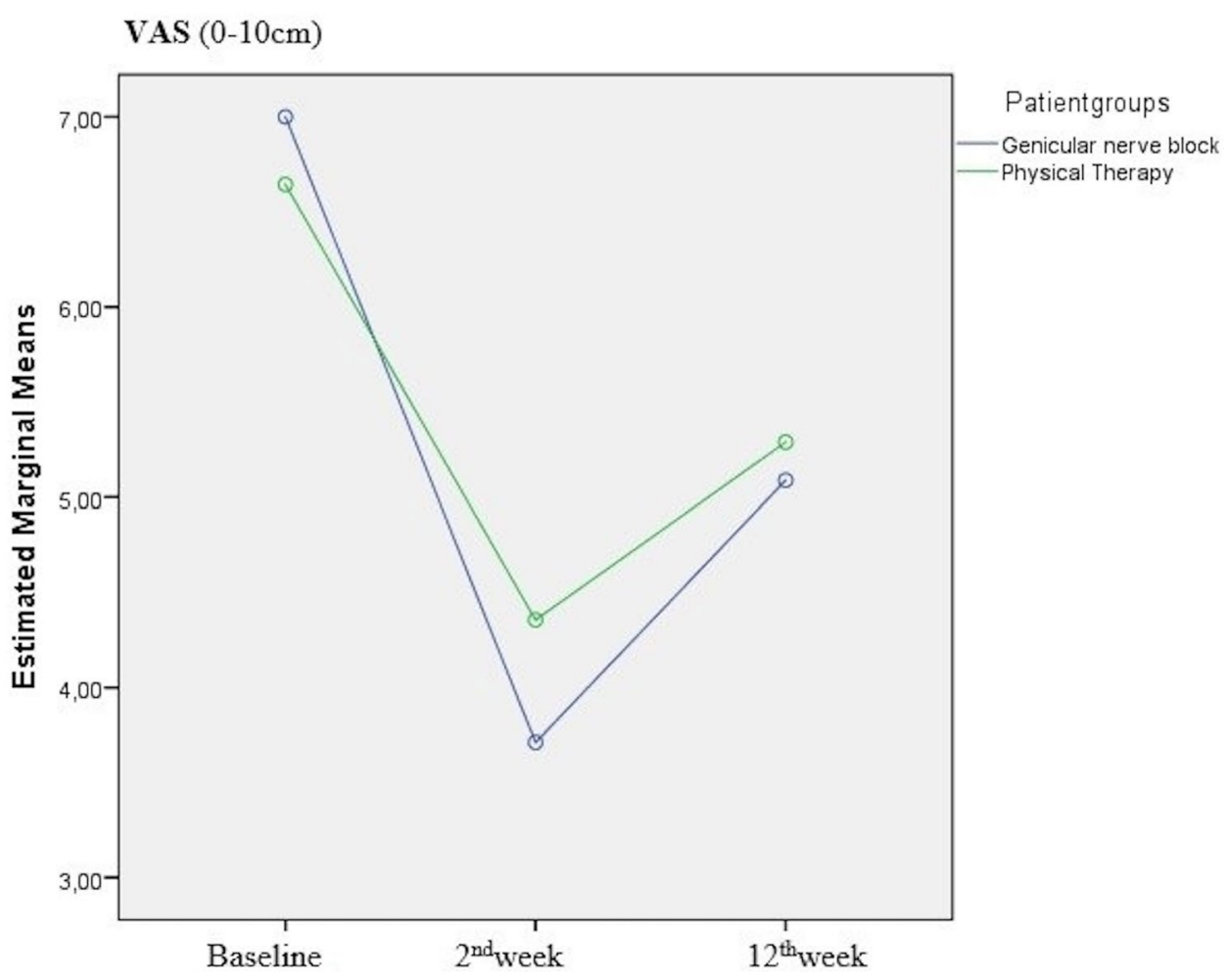

Fig. 5 Improvement in the

Western Ontario and McMaster Universities (WOMAC) scores

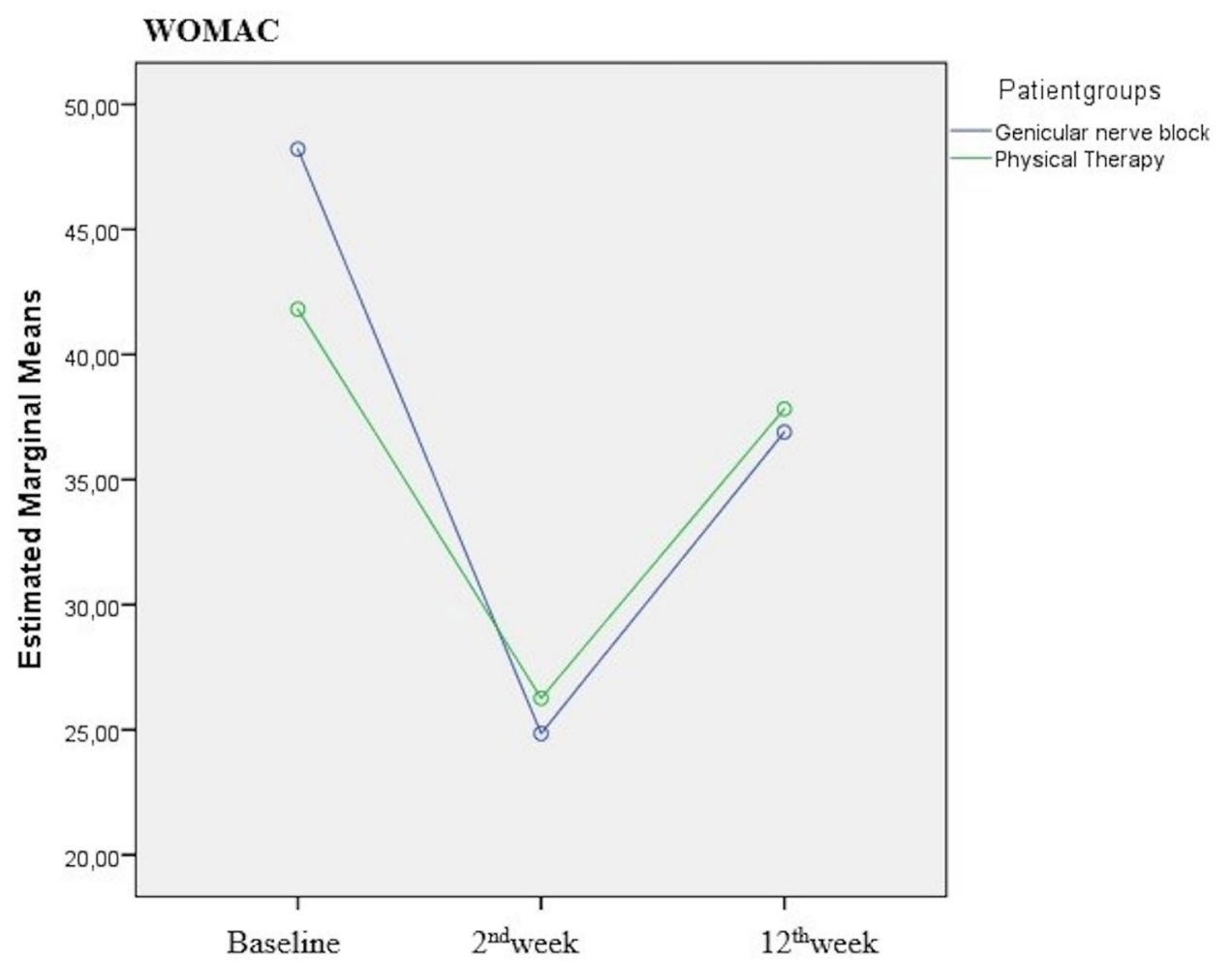


Fig. 6 Improvement in 6-min walking test scores

\section{6 minutes walking test}

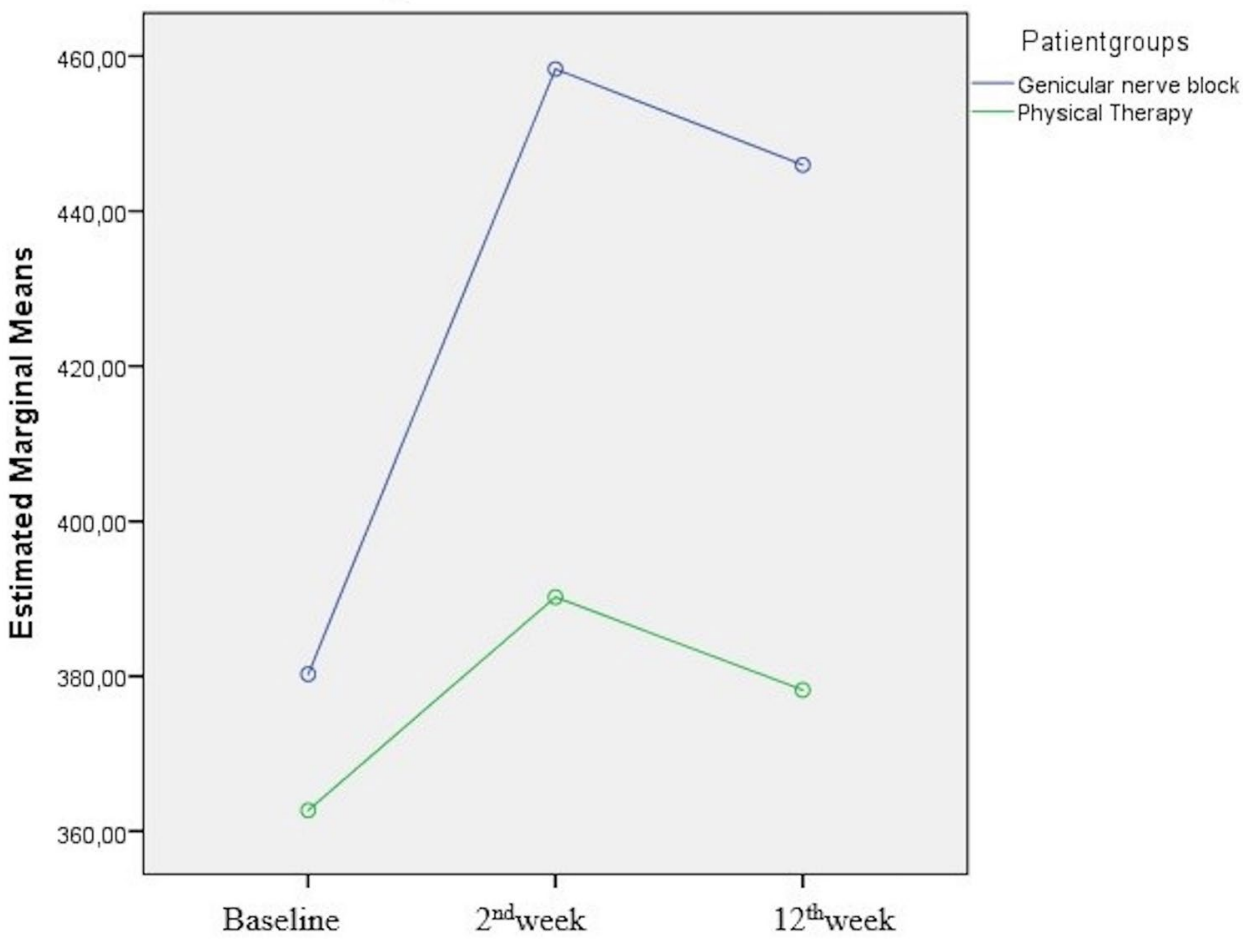

Table 1 Demographic and Clinical Variables at Baseline

\begin{tabular}{|c|c|c|c|c|}
\hline Variables & All patients $(n=102)$ & Genicular block $(n=51)$ & Physical therapy $(n=51)$ & $p$ value \\
\hline Age, (year) mean \pm SD (median; min-max) & $54.45 \pm 8.56(54 ; 41-80)$ & $55.88 \pm 7.62(54 ; 45-80)$ & $53.01 \pm 9.22(53 ; 41-72)$ & 0.077 \\
\hline \multicolumn{5}{|l|}{ Gender, $n(\%)$} \\
\hline Female & 81 & $40(78.4)$ & $41(80.4)$ & \multirow[t]{2}{*}{0.807} \\
\hline Male & 21 & $11(21.6)$ & $10(19.6)$ & \\
\hline \multicolumn{5}{|l|}{ Educational status $n(\%)$} \\
\hline Illiterate & $4(3.9)$ & $4(7.8)$ & 0 & \multirow[t]{5}{*}{0.322} \\
\hline Primary school & $39(38.2)$ & $18(35.3)$ & $21(40.2)$ & \\
\hline High school & $45(44.1)$ & $22(43.1)$ & $23(45.1)$ & \\
\hline University & $11(10.8)$ & $5(9.8)$ & $6(11.8)$ & \\
\hline Postgraduate & $3(2.9)$ & $2(3.9)$ & $1(2.0)$ & \\
\hline \multicolumn{5}{|l|}{ Marrital status, $n(\%)$} \\
\hline Married & 82 & $42(82.4)$ & $40(78.4)$ & \multirow[t]{3}{*}{0.026} \\
\hline Single & 9 & $7(13.7)$ & $2(3.9)$ & \\
\hline Widowed & 11 & $2(3.9)$ & $9(17.6)$ & \\
\hline \multicolumn{5}{|l|}{ Kellgren-Lawrence } \\
\hline Grade 2 & $65(63.7)$ & $32(62.7)$ & $33(64.7)$ & \multirow[t]{2}{*}{0.837} \\
\hline Grade 3 & $37(36.3)$ & $19(37.3)$ & $18(35.3)$ & \\
\hline Co-morbid diseases & $70(68.6)$ & $38(74.5)$ & $32(62.7)$ & 0.200 \\
\hline $\mathrm{BMI}$, mean $\pm \mathrm{SD}$ & $29.86 \pm 4.33$ & $30.94 \pm 4.02$ & $28.78 \pm 4.39$ & 0.059 \\
\hline VAS, mean \pm SD (median; min-max) & $6.87 \pm 1.81(7 ; 1-10)$ & $6.90 \pm 1.62(7 ; 1-10)$ & $6.84 \pm 1.89(7 ; 2-10)$ & 0.85 \\
\hline WOMAC, mean \pm SD & $46.31 \pm 18.81$ & $48.21 \pm 19.27$ & $43.18 \pm 17.89$ & 0.188 \\
\hline Pain & $9.93 \pm 4.09$ & $10.19 \pm 4.51$ & $9.30 \pm 3.80$ & 0.476 \\
\hline Stiffness (median; min-max) & $3.88 \pm 2.29(4 ; 0-9)$ & $3.09 \pm 2.08(4 ; 0-8)$ & $4.61 \pm 1.60(4 ; 2-9)$ & 0.012 \\
\hline Physical activity & $31.72 \pm 12.98$ & $33.73 \pm 13.43$ & $27.74 \pm 12.92$ & 0.069 \\
\hline $6 \mathrm{MWT}$ & $365.26 \pm 104.56$ & $369.25 \pm 105.33$ & $361.27 \pm 105.33$ & 0.457 \\
\hline
\end{tabular}

SD standard deviation, VAS Visual Analogue Scale, WOMAC Western Ontario and McMaster Universities Osteoarthritis Index, 6-MWT 6-min walking test 
Table 2 Changes in clinical outcomes

\begin{tabular}{|c|c|c|c|c|}
\hline \multirow[t]{2}{*}{ Clinical outcomes } & \multicolumn{2}{|c|}{ Genicular block $(n=40)$} & \multicolumn{2}{|c|}{ Physical therapy $(n=46)$} \\
\hline & Mean \pm SD & $p$ & Mean \pm SD & $p$ \\
\hline \multicolumn{5}{|l|}{ VAS } \\
\hline Baseline & $7.01 \pm 1.36$ & & $6.64 \pm 1.99$ & \\
\hline 2nd week & $3.71 \pm 2.18$ & $<0.001$ & $4.35 \pm 1.09$ & $<0.001$ \\
\hline 12 th week & $5.08 \pm 2.22$ & $<0.001$ & $5.25 \pm 1.33$ & $<0.001$ \\
\hline \multicolumn{5}{|l|}{ WOMAC-Total } \\
\hline Baseline & $48.02 \pm 19.33$ & & $41.18 \pm 17.69$ & \\
\hline 2nd week & $24.85 \pm 17.04$ & $<0.001$ & $26.26 \pm 10.20$ & $<0.001$ \\
\hline 12 th week & $36.91 \pm 16.91$ & $<0.001$ & $37.82 \pm 13.91$ & 0.001 \\
\hline \multicolumn{5}{|l|}{ WOMAC-Pain } \\
\hline Baseline & $10.19 \pm 4.51$ & & $9.30 \pm 3.80$ & \\
\hline 2nd week & $4.69 \pm 3.41$ & $<0.001$ & $4.15 \pm 1.43$ & $<0.001$ \\
\hline 12 th week & $7.85 \pm 4.51$ & $<0.001$ & $4.84 \pm 1.44$ & $<0.001$ \\
\hline \multicolumn{5}{|l|}{ WOMAC-Stiffness } \\
\hline Baseline & $3.09 \pm 2.08$ & & $4.61 \pm 1.60$ & \\
\hline 2nd week & $1.38 \pm 1.48$ & $<0.001$ & $3.38 \pm 1.47$ & $<0.001$ \\
\hline 12th week & $2.04 \pm 1.90$ & $<0.001$ & $4.73 \pm 1.48$ & 0.513 \\
\hline \multicolumn{5}{|c|}{ WOMAC-Physical activity } \\
\hline Baseline & $33.73 \pm 13.43$ & & $27.74 \pm 12.92$ & \\
\hline 2nd week & $18.75 \pm 13.03$ & $<0.001$ & $18.10 \pm 7.16$ & $<0.001$ \\
\hline 12 th week & $26.56 \pm 12.76$ & $<0.001$ & $28.45 \pm 11.89$ & 0.691 \\
\hline \multicolumn{5}{|l|}{$6 \mathrm{MWT}$} \\
\hline Baseline & $380.26 \pm 101.29$ & & $362.68 \pm 102.38$ & \\
\hline 2nd week & $458.33 \pm 139.99$ & $<0.001$ & $390.21 \pm 87.99$ & 0.001 \\
\hline 12th week & $445.95 \pm 139.65$ & 0.354 & $378.22 \pm 79.89$ & 0.025 \\
\hline
\end{tabular}

$S D$ standard deviation, VAS Visual Analogue Scale, WOMAC Western Ontario and McMaster Universities Osteoarthritis Index, 6-MWT 6-min walking test of age, gender, education level and BMI. Sociodemographic characteristics and Kellgren-Lawrence scores of the patients are presented in Table 1. There was no statistically significant difference between the two groups.

A comparison of the VAS, WOMAC and 6MWT scores between the groups is shown in Table 2. Improvement in all parameters was observed at both 2 and 12 weeks after the treatment in both groups (Figs. 4, 5 and 6). We observed no statistically significant difference in the change in VAS and WOMAC scores between the two groups.

The within-group comparison for all clinical variables before and after treatment is shown in Table 3. While the increase in the 6MWT score at the 2nd week was similar between the groups $(p=0.073)$, the 6MWT scores in the ultrasound-guided GNB group were higher at the 12th week $(p=0.046)$.

No side effects were observed in either study group.

\section{Discussion}

This study aimed to compare the effectiveness of ultrasoundguided GNB and PT for chronic KOA. We observed that ultrasound-guided GNB provides comparable benefit as PT on pain and knee function at 2 weeks and 12 weeks after treatment. Additionally, the improvement in gait (6MWT) was sustained for up to 12 weeks and lasted longer than the PT group.

Multiple studies have reported improvement in pain and function with PT for KOA [10, 21-24]. On the other hand, ultrasound-guided GNB is an emerging treatment option that aims to control pain by inhibiting knee innervation. A recent study comparing ultrasound-guided versus blinded GNB injections revealed the superiority of ultrasoundguided injections in improving the functionality in KOA patients [25]. Thus, our functional improvement findings 
Table 3 Intergroup comparison of clinical variables before and after treatment

\begin{tabular}{lccc}
\hline Variables & $\begin{array}{l}\text { Genicular Block }(n=40) \\
\text { Mean difference } \pm \text { SD }\end{array}$ & $\begin{array}{l}\text { Physical therapy }(n=46) \\
\text { Mean difference } \pm \text { SD }\end{array}$ & $p$ \\
\hline VAS & & & \\
Baseline-2nd week & $3.28 \pm 0.32$ & $1.35 \pm 0.13$ & 0.652 \\
Baseline-12th week & $1.91 \pm 0.31$ & & 0.823 \\
WOMAC & & $15.55 \pm 2.54$ & 0.264 \\
Baseline-2nd week & $23.36 \pm 3.31$ & $3.99 \pm 1.90$ & 0.491 \\
Baseline-12th week & $11.31 \pm 2.57$ & & 0.329 \\
WOMAC-pain & & $5,15 \pm 0,62$ & 0.059 \\
Baseline-2nd week & $5.51 \pm 0,75$ & $4.46 \pm 0.63$ & 0.063 \\
Baseline-12th week & $2,33 \pm 0,63$ & & 0.001 \\
WOMAC-stiffness & $1.74 \pm 0,33$ & $1.23 \pm 0.32$ & \\
Baseline-2nd week & $1.04 \pm 0.31$ & $-0.11 \pm 2.68$ & 0.205 \\
Baseline-12th week & & & 0.467 \\
WOMAC-physical activity & $14.98 \pm 2.26$ & $9.64 \pm 1.85$ & \\
Baseline-2nd week & $7.17 \pm 1.77$ & $-0.71 \pm 1.76$ & 0.073 \\
Baseline-12th week & & & 0.41 \\
6 MWT & $78.06 \pm 14.75$ & $27.53 \pm 6.55$ & $15.53 \pm 6.68$ \\
Baseline-2nd week & $65.68 \pm 15.75$ & & \\
Baseline-12th week & & & \\
\hline
\end{tabular}

$S D$ standard deviation, VAS Visual Analogue Scale, WOMAC Western Ontario and McMaster Universities Osteoarthritis Index, 6-MWT 6-min walking test can also be attributed to precision attained by the ultrasound-guided administration of the anaesthetic solution.

Demir et al. [26] reported a patient with KOA who was previously unresponsive to conservative therapies and showed dramatic improvements in the VAS and WOMAC scores at 4 weeks (baseline: $80 \mathrm{~mm}$ and 96 and posttreatment: $10 \mathrm{~mm}$ and 5, respectively) and persisting up to 24 weeks ( $0 \mathrm{~mm}$ and 4 respectively) when treated with ultrasound-guided GNB. We also observed pronounced improvement in these scores at the 2 weeks follow-up; however, unlike their study, we observed a slight increase in the scores between the 2 post-treatment assessments. This is possible since their result was based on a single patient, whereas, we had a sufficiently large study sample. In addition, physical capacity was also evaluated in our study, and the increase in the 6-min walking test continued until the 12th week, which is a remarkable result of our study. We believe that the findings of our study would contribute to further studies and literature about the procedure.

Yilmaz et al. [16] compared GNB under ultrasound guidance with and without additional intra-articular corticosteroid injection in patients with KOA. Unlike our study, they used a local anaesthetic as GNB solution and the corticosteroid solution was injected intra-articular and observed significant improvements in pain and function in both groups. Other authors have also reported that combining corticosteroids with a local anaesthetic provides a long-term analgesic effect [27]. Nevertheless, we were able to obtain comparable results without intra-articular administration of corticosteroid, and this approach may have protected us from iatrogenic concerns, such as the risk of septic arthritis.

In a recent study by Elsaman et al. [13], GNB and intraarticular triamcinolone were injected in patients with rheumatoid arthritis, and GNB was shown to be effective not only in improving pain and function but also in reducing inflammation. They also noted that the decrease in the power Doppler score continued till the 12 th week in the GNB group, but there was a rapid rebound effect seen in the intra-articular injection group and the power Doppler score increased again at the 12 th week. Therefore, it can be deduced that GNB may be a potential treatment option for inflammatory arthritis.

This study has some limitations. First is the lack of a control group. However, we tried to address this limitation by providing a standard home exercise programme to both groups besides their respective group intervention. Secondly, exercise compliance was assessed only by patients' verbal confirmation. It would have been more appropriate to carry out their exercise session in a hospital setting under supervision. Lastly, the follow-up period was relatively short. Therefore, there is a need for further studies examining long-term effectiveness of ultrasoundguided GNB on pain and function. 


\section{Conclusions}

Our results prove that pain reduction and functional improvement by PT and ultrasound-guided GNB are comparable, however, the increase in physical capacity is greater with ultrasound-guided GNB persisting up to 12 weeks. Considering the frequency of treatment sessions and the length of hospital stay, ultrasound-guided GNB can be employed before PT, keeping in mind the current scenario of healthcare service during the COVID-19 pandemic.

Acknowledgements The authors would like to thank Enago (www. enago.com) for the English language review.

\section{References}

1. Fibel KH, Hillstrom HJ, Halpern BC (2015) State-of-the-art management of knee osteoarthritis. World J Clin Cases 3:89-101. https://doi.org/10.12998/wjcc.v3.i2.89

2. Sert AT, Sen EI, Esmaeilzadeh S, Ozcan E (2020) The effects of dextrose prolotherapy in symptomatic knee osteoarthritis: a randomized controlled study. J Altern Complement Med 26:409-417. https://doi.org/10.1089/acm.2019.0335

3. Cross M, Smith E, Hoy D, Nolte S, Ackerman I, Fransen M et al (2014) The global burden of hip and knee osteoarthritis: estimates from the global burden of disease 2010 study. Ann Rheum Dis 73:1323-1330

4. Kidd VD, Strum SR, Strum DS, Shah J (2019) Genicular nerve radiofrequency ablation for painful knee arthritis: the why and the how. JBJS Essent Surg Tech 13(9):e10

5. Deyle GD, Allison SC, Matekel RL et al (2005) Physical therapy treatment effectiveness for osteoarthritis of the knee: a randomized comparison of supervised clinical exercise and manual therapy procedures versus a home exercise program. Phys Ther 85:1301-1317

6. van Doormaal MCM, Meerhoff GA, Vliet Vlieland TPM, Peter WF (2020) A clinical practice guideline for physical therapy in patients with hip or knee osteoarthritis. Musculoskeletal Care 18:575-595

7. Dantas LO, Salvini TF, McAlindon TE (2021) Knee osteoarthritis: key treatments and implications for physical therapy. Braz J Phys Ther 25:135-146

8. Kesikburun S, Yaşar E, Uran A, Adigüzel E, Yilmaz B (2016) Ultrasound-guided genicular nerve pulsed radiofrequency treatment for painful knee osteoarthritis: a preliminary report. Pain Phys 19:E751-759

9. Choi WJ, Hwang SJ, Song JG, Leem JG, Kang YU, Park PH et al (2011) Radiofrequency treatment relieves chronic knee osteoarthritis pain: a double-blind randomized controlled trial. Pain 152:481-487

10. McCormick ZL, Korn M, Reddy R, Marcolina A, Dayanim D, Mattie R, Cushman D, Bhave M, McCarthy RJ, Khan D, Nagpal G, Walega DR (2017) Cooled radiofrequency ablation of the genicular nerves for chronic pain due to knee osteoarthritis: sixmonth outcomes. Pain Med 18:1631-1641. https://doi.org/10. 1093/pm/pnx069
11. Billesberger LM, Fisher KM, Qadri YJ, Boortz-Marx RL (2020) Procedural treatments for knee osteoarthritis: a review of current injectable therapies. Pain Res Manag. https://doi.org/10.1155/ 2020/3873098

12. Kim DH, Lee MS, Lee S, Yoon SH, Shin JW, Choi SS (2019) A prospective randomized comparison of the efficacy of ultrasound- vs fluoroscopy-guided genicular nerve block for chronic knee osteoarthritis. Pain Phys 22:139-146

13. Elsaman AM, Maaty A, Hamed A (2021) Genicular nerve block in rheumatoid arthritis: a randomized clinical trial. Clin Rheumatol 40:4501-4509

14. Altman RD, Bloch DA, Bole GG Jr et al (1987) Development of clinical criteria for osteoarthritis. J Rheumatol 14:3-6

15. Kellgren JH, Lawrence JS (1957) Radiological assessment of osteo-arthrosis. Ann Rheum Dis 16:494-502

16. Yilmaz V, Umay E, Gundogdu I, Aras B (2021) The comparison of efficacy of single intraarticular steroid injection versus the combination of genicular nerve block and intraarticular steroid injection in patients with knee osteoarthritis: a randomised study. Musculoskelet Surg 105:89-96

17. Altaş EU, Demirdal Ü (2020) The effect of physical therapy and rehabilitation modalities on sleep quality in patients with primary knee osteoarthritis: a single-blind, prospective, randomized-controlled study. Turk J Phys Med Rehabil 3(66):73-83

18. Boonstra AM, Schiphorst Preuper HR, Reneman MF, Posthumus JB, Stewart RE (2008) Reliability and validity of the visual analogue scale for disability in patients with chronic musculoskeletal pain. Int J Rehabil Res 31:165-169

19. Basaran S, Guzel R, Seydaoglu G, GulerUysal F (2010) Validity, reliability, and comparison of the WOMAC osteoarthritis index and Lequesne algofunctional index in Turkish patients with hip or knee osteoarthritis. Clin Rheumatol 29:749-756

20. Guyatt GH, Sullivan MJ, Thompson PJ, Fallen EL, Pugsley SO, Taylor DW et al (1985) The 6-minute walk: a new measure of exercise capacity in patients with chronic heart failure. Can Med Assoc J 132:919-923

21. Sun E, Moshfegh J, Rishel CA, Cook CE, Goode AP, George SZ (2018) Association of early physical therapy with long-term opioid use among opioid-naïve patients with musculoskeletal pain. JAMA Netw Open 1(8):e185909

22. Jamtvedt G, Dahm KT, Christie A et al (2008) Physical therapy interventions for patients with osteoarthritis of the knee: an overview of systematic reviews. Phys Ther 88:123-136

23. Khoja SS, Almeida GJ, Freburger JK (2020) Recommendation rates for physical therapy, lifestyle counseling and pain medications for managing knee osteoarthritis in ambulatory care settings: cross-sectional analysis of the National Ambulatory Care Survey (2007-2015). Arthritis Care Res (Hoboken) 72:184-192

24. Deyle GD, Allen CS, Allison SC, Gill NW, Hando BR, Petersen EJ, Dusenberry DI, Rhon DI (2020) Physical therapy versus glucocorticoid injection for osteoarthritis of the knee. N Engl J Med 382:1420-1429

25. Cankurtaran D, Karaahmet OZ, Yildiz SY, Eksioglu E, Dulgeroglu D, Unlu E (2020) Comparing the effectiveness of ultrasound guided versus blind genicular nerve block on pain, muscle strength with isokinetic device, physical function and quality of life in chronic knee osteoarthritis: a prospective randomized controlled study. Korean J Pain 33:258-266

26. DemIr Y, Güzelküçük Ü, Tezel K, AydemIr K, Taşkaynatan MA (2017) A different approach to the management of osteoarthritis in the knee: ultrasound guided genicular nerve block. Pain Med 18:181-183 
27. An K, Elkassabany NM, Liu J (2015) Dexamethasone as adjuvant to bupivacaine prolongs the duration of thermal antinociception and prevents bupivacaine-induced rebound hyperalgesia via regional mechanism in a mouse sciatic nerve block model. PLoS ONE 10:e123459
Publisher's Note Springer Nature remains neutral with regard to jurisdictional claims in published maps and institutional affiliations. 